\title{
Effects of Retinoic Acid on Proliferation and Differentiation of HepG2 Cells
}

\author{
Michelle R. Burley ${ }^{1,3}$ and Charles M. Roth ${ }^{*}, 1,2,3$ \\ ${ }^{1}$ Department of Chemical and Biochemical Engineering, ${ }^{2}$ Department of Biomedical Engineering, and ${ }^{3}$ Graduate \\ Program in Molecular BioSciences, Rutgers, The State University of New Jersey, USA
}

\begin{abstract}
Hepatoma cell lines have characteristics derived from both their hepatic lineage and their transformation. We examined the potential of retinoic acid to enhance the function of a hepatoma cell line, HepG2, under conditions of reduced serum as are desired for bioreactor cultivation. We found that retinoic acid drastically slows the growth of HepG2 cells and induces a more spread morphology. Retinoic acid also augments the differentiated function of the cells, as measured by albumin and urea secretion rates. Expression levels of a panel of liver-enriched transcription factor were increased from retinoic acid exposure. Overall, we demonstrate that retinoic acid has significant effects on HepG2 growth and differentiated function. These results have implications for the use of retinoic acid both as a chemotherapeutic agent and as a medium component for cells of hepatic origin.
\end{abstract}

Keywords: Hepatocyte culture, differentiated function, liver-enriched transcription factors, gene expression.

\section{INTRODUCTION}

Hepatoma cells are useful in understanding both phenotypes of liver cancer and phenotypes of hepatocytes relevant to healthy and diseased states. Among human hepatoma cell lines, HepG2 is probably the best characterized. HepG2 cells serve a variety of functions in the laboratory, including assessment of the toxicology of compounds, such as antiviral drugs, genotoxicants and antigenotoxic agents [1-4]. HepG2 cells are also employed to study a range of physiological and pathophysiological conditions, including liver disease, gene expression and transcription in cancer, inflammatory response, and blood coagulation [5-8]. Furthermore, HepG2 cells are being developed as a potential cell source for bioartificial liver devices [9]. Their versatility and function make them extremely useful as a model system for liver function.

The liver is one of a few organs in the body capable of proliferation and differentiation when necessary in response to trauma, stress, or during homeostasis. There is often a balance between differentiation and proliferation, with cells ending their proliferation cycle through differentiation. However, when taken from an animal and placed into culture, primary hepatocytes do not proliferate and tend to exhibit decreased levels of differentiated functions. In contrast, HepG2 cells are capable of both proliferation and differentiation, which enhances their utility.

In the present study, we investigated the potential of retinoic acid to influence HepG2 growth, morphology, function, and gene expression. Retinoic acid, a derivative of vitamin A, plays many roles in cell differentiation, proliferation, and apoptosis. Retinoic acid has been shown to cause growth arrest and differentiation in several cancer cell lines [9-14]. Retinoic acid has previously been determined to slow HepG2 growth $[15,16]$, and high concentrations $(166 \mu \mathrm{M})$, induced

*Address correspondence to this author at the Department of Biomedical Engineering, 599 Taylor Road, Piscataway, NJ 08854, USA; Tel: 732-4454500x6205; Fax: 732-445-3753; E-mail: cmroth@rci.rutgers.edu apoptosis [17]. We show that significantly lower concentrations $(1-10 \mu \mathrm{M})$ are sufficient to quantitatively alter morphology, proliferation, gene expression, and levels of hepatic differentiation markers under conditions of reduced serum in the culture medium, which is particularly advantageous for HepG2 cell cultivation in bioreactors.

\section{MATERIALS AND METHODOLOGY}

Cell culture. Human HepG2 cells (ATCC, Rockville, Maryland) were maintained in MEM medium supplemented with 10\% fetal bovine serum (FBS) (Invitrogen), $200 \mathrm{U} / \mathrm{ml}$ penicillin, $200 \mu \mathrm{g} / \mathrm{ml}$ streptomycin, $100 \mu \mathrm{M}$ non-essential amino acids, $1 \mathrm{mM}$ MEM sodium pyruvate, and $2 \mathrm{mM} \mathrm{L-}$ glutamine (Invitrogen) in a humidified $37^{\circ} \mathrm{C}, 5 \% \mathrm{CO}_{2}$ incubator. Cells were plated at an initial density of $1 \times 10^{5}$ cells $/ \mathrm{cm}^{2}$ in $10 \%$ FBS media overnight. Cells were then grown for 14 days in MEM media supplemented with $2 \%$ FBS, with an equivalent volume of serum replacement solution containing $3 \mathrm{mg} / \mathrm{ml}$ bovine serum albumin (Sigma) and $315 \mathrm{mOsm} / \mathrm{kg} \mathrm{NaCl}$ (Sigma), in the presence or absence of $10,5,2.5$, or $1 \mu \mathrm{M}$ trans-retinoic acid (tRA) (Sigma). The tRA was diluted from a stock concentration of $100 \mathrm{mM}$ in DMSO (Sigma).

Image analysis. Images were taken on an Olympus CK40 at $20 \mathrm{X}$ magnification. Areas and aspect ratios were calculated using Olympus MicroSuite program with a sample size of 50 cells.

Real-time PCR. On days 7 and 14 of the experiment, cell samples were taken and RNA extracted using the Roche total RNA extraction kit (Roche). The cDNA was created using specific primers and AMV reverse transcriptase (Promega) according to the manufacturer's directions. Real-time PCR was performed using a Lightcycler (Roche) and QuantiTech SYBR Green PCR kit (Qiagen). RNA levels were normalized using $18 \mathrm{~S}$ rRNA as a stable housekeeping marker. For a particular gene, mRNA levels were calculated using the formula mRNA $_{\text {gene }}=2^{\wedge}\left(\mathrm{C}_{\mathrm{T}, 18 \mathrm{~S}}-\mathrm{C}_{\mathrm{T} \text {, gene }}\right)$, where $\mathrm{C}_{\mathrm{T}}$ is the "crossing point" or (interpolated) cycle at which the fluorescence reaches a predetermined value. The mRNA level for each 
gene in each sample was then normalized within each experiment to that gene in a control sample.

Metabolic Secretory Assays. Albumin secretion rates in culture supernatants were detected via competitive ELISA using purified human albumin standards (MP Biomedicals) and peroxidase-conjugated secondary antibody for detection (MP Biomedicals) in accordance with previous protocols [18]. Urea secretion rates were measured from supernatants using an enzymatic urea nitrogen kit (Stanbio). Data were obtained in triplicates and fit to standards using either a semi-logarithmic (albumin) or a linear (urea) curve. These values were then normalized to the numbers of viable cells for each sample.

Statistics. Samples were of three separate experiments with three replicates each. Samples from each set were normalized to those for control, and the average was taken across experiments. Errors represent the standard error of the mean, and comparisons among groups were made by ANOVA, followed by Tukey's test for individual comparisons.

\section{RESULTS}

In order to observe both growth and differentiation effects of retinoic acid, we seeded HepG2 cells at a moderate density of $1.0 \times 10^{5}$ cells $/ \mathrm{cm}^{2}$ on 6 well plates in $10 \% \mathrm{FBS}$ media and allowed them to attach overnight. Cells were then grown for 14 days in reduced serum ( $2 \%$ FBS) media, containing 0-10 $\mu \mathrm{M}$ all-trans retinoic acid (tRA), DMSO (which is the solvent for tRA), or untreated as a control. We observed the morphological and functional characteristics over a 14 day period following onset of treatment. Retinoic acid treatment induces several morphological changes, with cells becoming larger, and in some cases, multinucleated, at all of the tRA concentrations tested (data not shown). In comparison, DMSO and control cells maintain small size with a single nucleus per cell. Quantitative analysis of the cells shows that tRA treated cells are statistically larger than the corresponding controls and that the enlargement is not due to elongation, since the cell aspect ratio is not significantly altered by tRA treatment (Table 1).

Table 1. Cell Morphological Characteristics

\begin{tabular}{|c|c|c|}
\hline Treatment & Aspect Ratio & Area $\left(\mu \mathbf{~ m}^{2}\right)$ \\
\hline \hline Untreated & $1.64 \pm 0.06$ & $427 \pm 21$ \\
\hline DMSO & $1.50 \pm 0.05$ & $419 \pm 16$ \\
\hline $1 \mu \mathrm{M}$ tRA & $1.66 \pm 0.06$ & $970 \pm 50\left(^{*}\right)$ \\
\hline $2.5 \mu \mathrm{M}$ tRA & $1.55 \pm 0.04$ & $1260 \pm 178\left(^{*}\right)$ \\
\hline $5 \mu \mathrm{M}$ tRA & $1.59 \pm 0 . .05$ & $1061 \pm 52\left(^{*}\right)$ \\
\hline $10 \mu \mathrm{M}$ tRA & $1.61 \pm 0.05$ & $1249 \pm 81\left(^{*}\right)$ \\
\hline${ }^{*} \mathrm{p}<0.05 v s$ untreated control. &
\end{tabular}

We then examined the effect of tRA on growth. Consistent with behavior observed in 10\% FBS [16], retinoic acid retards the growth of HepG2 cells (Fig. 1) at all concentrations employed $(1-10 \mu \mathrm{M})$ in reduced $(2 \%)$ serum. However, growth in reduced serum is slowed markedly as compared to the corresponding conditions in $10 \%$ FBS [16]. The concentration of DMSO used as a solvent does not affect the morphology (Table 1) or cell growth (Fig. 1).

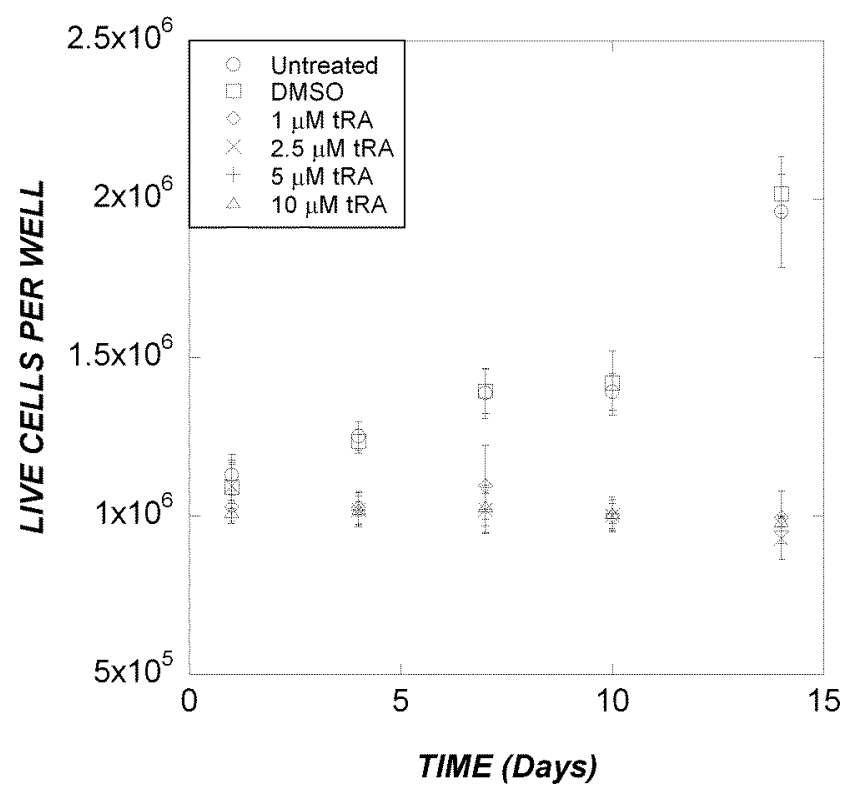

Fig. (1). Retinoic acid slows HepG2 cell growth. From days 4-14, all tRA treated samples are significantly different $(\mathrm{p}<0.05)$ as compared to DMSO or untreated controls.

Since we observed differences in the morphology and growth, we examined whether these were accompanied by changes in differentiated function. Albumin and urea represent two prototypical markers of protein synthetic and metabolic differentiated function in hepatocytes, and the effect of tRA on these hepatic outputs has not been investigated previously. We find that albumin levels are not affected by tRA treatment until day 10 , after which tRA treated cells produce markedly more albumin (Fig. 2). By day 14 the effect of tRA on albumin secretion is dose-dependent, with a maximum in the range of 2.5 to $5 \mu \mathrm{M}$. We have observed a similar biphasic response in the albumin secretion of primary rat hepatocytes exposed to tRA (Burley and Roth. Submitted for publication). HepG2 cells also exhibit an increase in urea output with tRA treatment, with significant differences observed beginning at day 4 and continuing though the 14 day course of the experiment (Fig. 3). The increase in urea production appeared to be weakly concentration dependent, with a slight maximum at $2.5 \mu \mathrm{M}$ at 4 days that dissipated at later times.

The changes in morphology, growth, and function led us to examine the gene expression levels, using real-time PCR, of several liver-enriched transcription factors associated with hepatic differentiated function. We first examined the levels of albumin transcripts to determine if mRNA levels corresponded to secretion rates. Albumin mRNA levels are repressed slightly with DMSO treated cells and are increased for 5 and $10 \mu \mathrm{M}$ tRA on days 7 and 14 respectively (Fig. 4A). This corresponds with increased albumin protein secretion on those days (Fig. 2). However, the magnitude of changes in albumin RNA levels were considerably less than the changes in secreted protein levels, suggesting that both transcriptional and post-translational processes may play a role in regulating increased albumin protein levels. 


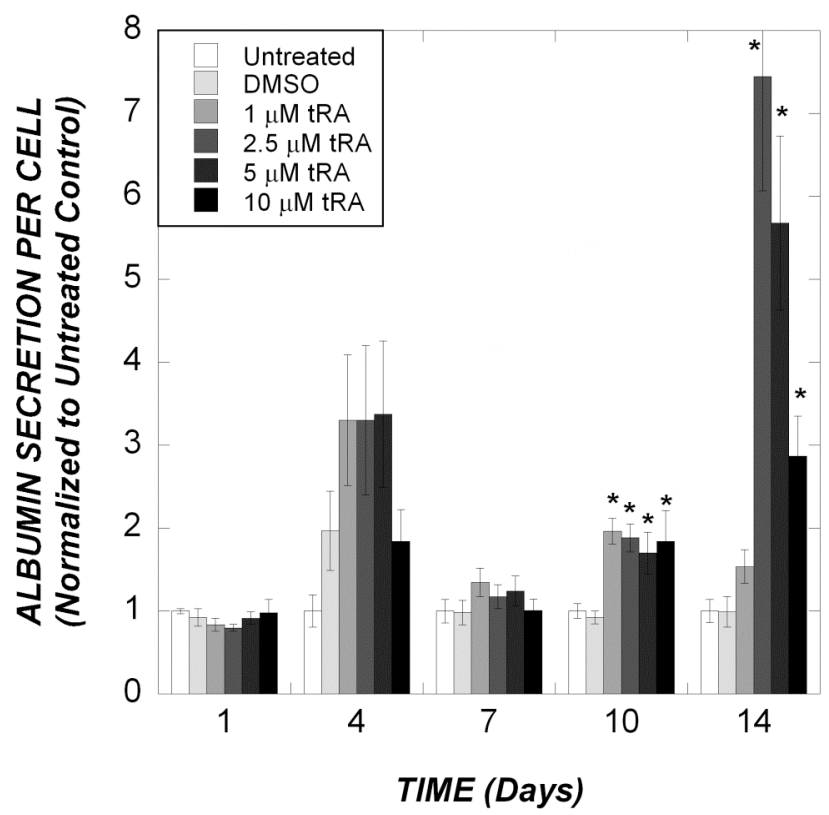

Fig. (2). Dose response effect of retinoic acid on albumin secretion. HepG2 cells were treated with varying concentrations of tRA over a 14 day period. Samples are normalized to untreated control. The symbols $(*)$ represent $\mathrm{p}<0.05$ sample versus untreated control.

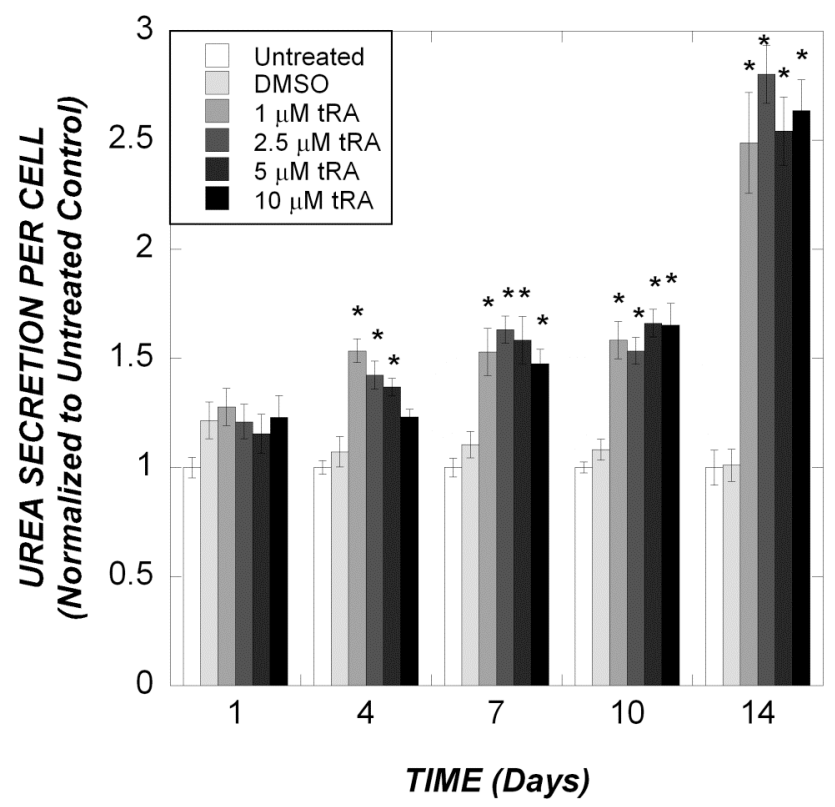

Fig. (3). Dose response effect of retinoic acid on urea secretion. HepG2 cells were treated with varying concentrations of tRA over a 14 day period. Samples are normalized to untreated control. The symbols $(*)$ represent $\mathrm{p}<0.05$ sample versus untreated control.

A panel of liver-enriched transcription factor genes that are expected to reflect the differentiated state of hepatocytes was profiled. Surprisingly, all of these genes were induced (upregulated) by tRA. However, their fold-change and dynamics varied considerably. The responses can be grouped into three categories: early, late, and continuous. The retinoic acid responsive genes that are induced early, as defined by high expression on day 7 and not day 14, are CEBP $\alpha$ (Fig.
4H), CEBP $\beta$ (Fig. 4I) and HNF1 $\alpha$ (Fig. 4B). HNF4 $\alpha$ (Fig. 4F) exhibited a transient response to DMSO at day 7, masking tRA effects but was induced relative to DMSO or untreated controls for all tRA levels at day 14. The genes that are responsive to retinoic acid on both days 7 and 14 are HNF1 $\beta$ (Fig. 4C), HNF3 $\alpha$ (Fig. 4D), HNF3 $\beta$ (Fig. 4E), and HNF6 $\alpha$ (Fig. 4G). Some of the inductions were dramatic, including HNF6 $\alpha$ with over a 70 fold increase for the 5 and $10 \mu \mathrm{M}$ tRA conditions, CEBP $\alpha$ with a dramatic increase for all retinoic acid conditions, and CEBP $\beta$ with an over 30 fold increase for the $10 \mu \mathrm{M}$ tRA condition.

\section{DISCUSSION}

The liver is an essential organ that regulates many metabolic processes. Liver diseases such as hepatitis A-C, liver cancer, and acute liver failure claim over 20,000 lives per year [19]. The best long-term solution for many of these patients is a liver transplant, but demand far outstrips supply. Liver assist devices, including bioartificial livers, can alleviate the problem, but there are many issues that limit development. One of the main issues is the type of cells being used in the bioartificial device. Primary human hepatocytes, the best cells for the task, are hard to come by and do not divide in vitro. Porcine hepatocytes and other xenogeneic cell lines, though relatively easy to acquire, are hampered by immunological problems and must be freshly isolated or cryopreserved with a concomitant loss in viablilty. Immortalized fetal or adult hepatocytes are another potential source, though they have limited function and division capability. Stem cells, from embryonic to hepatic, are an interesting alternative, but the ability to turn them into viable liver cells is still in progress.

Another approach is the use of human hepatoma cells lines, such as HepG2 [20]. One clinically tested liver assist device has used the $\mathrm{C} 3 \mathrm{~A}$ derivative of the HepG2 cell line [21]; however, this device did not result in improvements in overall survival or hepatic markers in phase I clinical trials. A major issue limiting the application of hepatoma cell lines is maintenance of differentiated function in liver assist devices. Furthermore, the ability to promote liver specific functions of HepG2 cells would aid in their application to toxicology screening and may be relevant in the context of hepatocarcinoma as well.

In the liver, there is a balance between differentiation and proliferation. Liver stem cells proliferate, then differentiate, in response to trauma, stress, or during normal development [22]. HepG2 cells recapitulate this balance, as they are capable of both proliferation and differentiation. There are several agents, such as trichostatin A, that may alter this balance by inducing cell cycle arrest and differentiation of HepG2 cells [23]. Such agents are of great interest due to their potential to prevent or slow tumorigenesis, but they could also be of great utility in the application of hepatoma cells for living cell devices, including bioartifical liver.

Derivatives of vitamin A, such as all-trans retinoic acid (tRA), play important roles in a variety of physiological processes, including vision, metabolism and differentiation [24]. Retinoic acid regulates the expression of many liver enriched genes, such as phosphoenolpyruvate carboxykinase (PEPCK), haptoglobin, and $\alpha-1$ acid glycoprotein [25-27]. We found that tRA promotes the production of albumin and urea, two mark- 
(A)

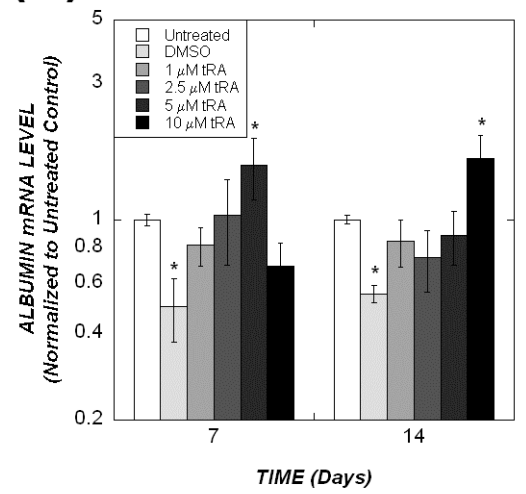

(D)

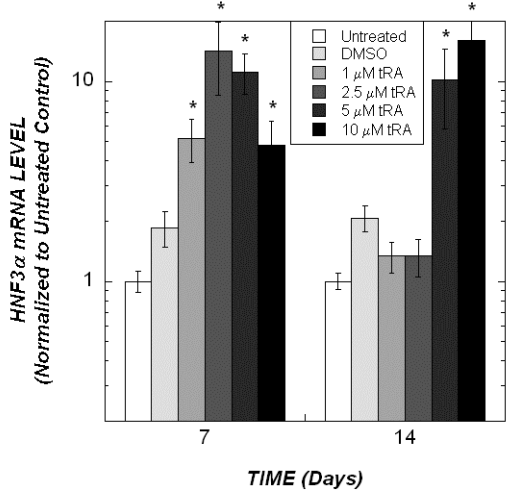

(G)

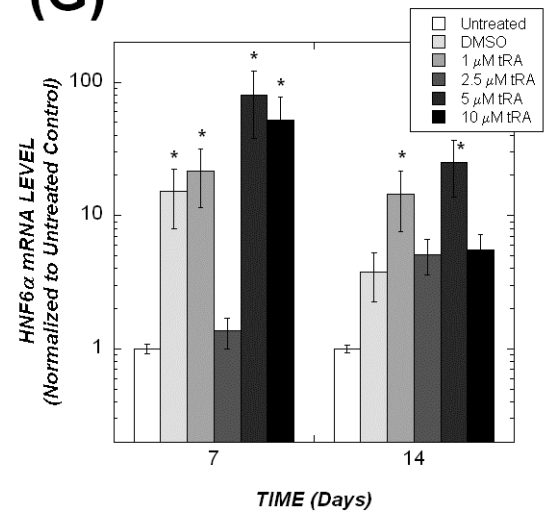

(B)

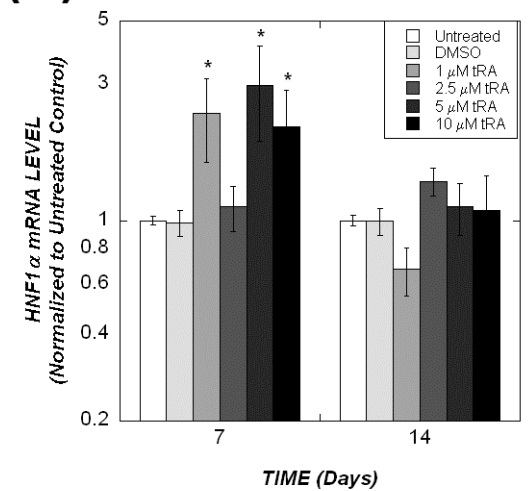

(E)

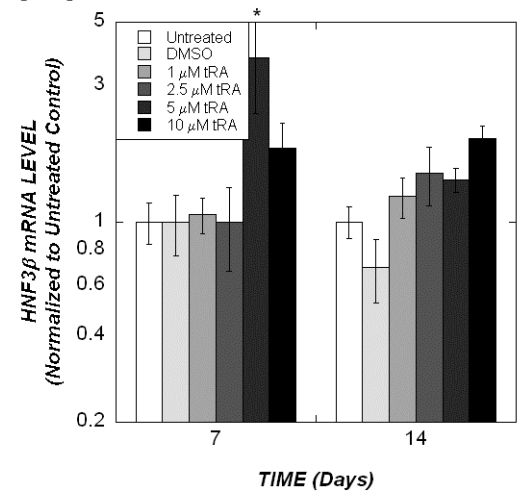

(H)

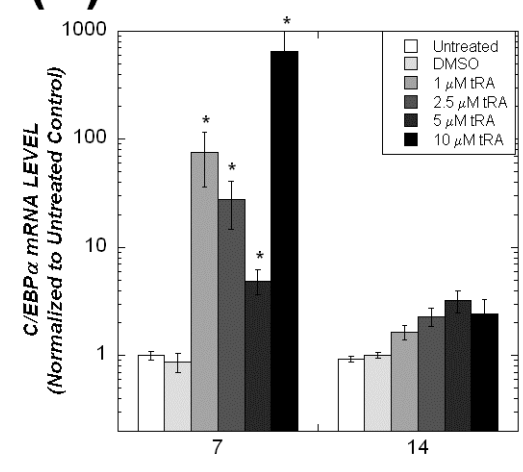

TIME (Days)
(C)

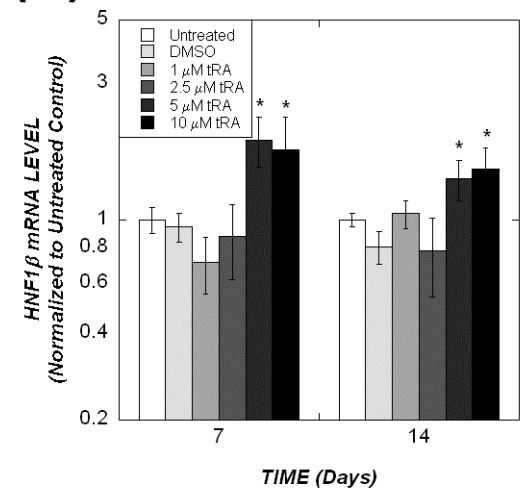

(F)

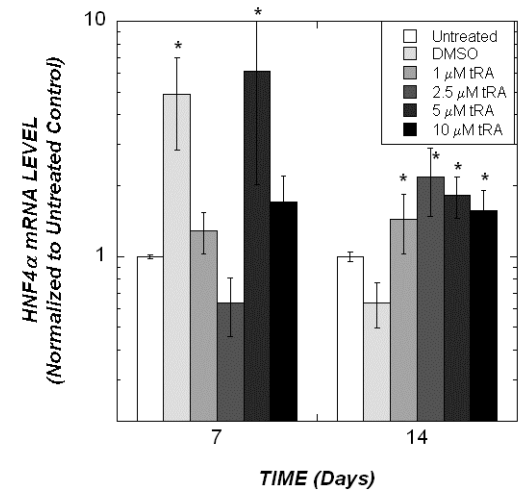

(I)

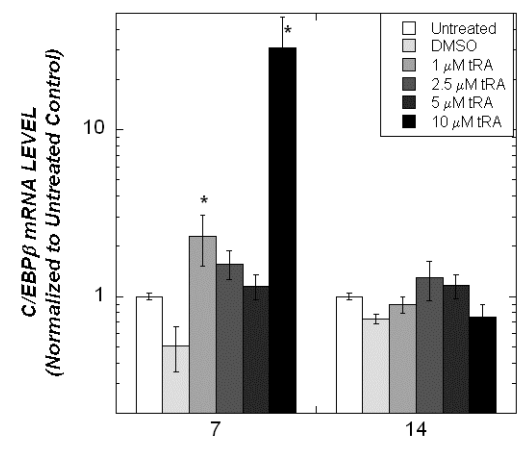

TIME (Days)

Fig. (4). Retinoic acid alters transcription factor levels. Albumin (A), HNF1 $\alpha$ (B), HNF1 $\beta$ (C), HNF3 $\alpha$ (D), HNF3 $\beta$ (E), HNF4 $\alpha(\mathbf{F})$, HNF6a (G), CEBPa (H), CEBP $\beta(\mathbf{I})$. The symbols $\left(^{*}\right)$ represent $\mathbf{p}<0.05$ sample versus untreated control.

ers of differentiation commonly used in the assessment of culture conditions for hepatocytes $[28,29]$. Taken together, these results imply that retinoic acid activates signaling pathways and/or transcriptional programs that are fundamental to the differentiated hepatic phenotype.

It has been shown previously that tRA induces growth arrest and morphological changes in HepG2 cells [16]. The mechanisms by which this occurs are unclear. Retinoic acid induces growth arrest of HepG2 cells possibly by altering the activities of cyclin-cdk complexes involved in G1/S transition and S-phase progression [15]. Alternatively, the dramatic increase in cell area observed in this study under tRA treatment implicates cell-matrix and cell-cell signaling as potential mechanisms that could lead to contact inhibition and alter the balance between proliferation and differentiation. For instance, tRA has been observed to reduce free pools of $\beta$-catenin [30], thus reducing proliferation signaling through the wnt pathway. Retinoic acid receptor $\alpha$ also has the capability to bind $\beta$ catenin, thus reducing the amount of TCF-4/ $\beta$-catenin complex, which is a proliferation signal [1]. Sequestration of $\beta$ catenin by E-cadherin has been shown to increase differentiated function of hepatocytes [31]; thus, tRA may work using a similar mechanism. This reduction in the proliferation signal may shift the balance towards differentiation. 
However, RA influences a number of cellular pathways, and as such, its mechanism of action is probably multi-faceted. Retinoic acid may improve differentiation signals by increasing the amounts and phosphorylation of connexins, their stabilization in plasma membrane plaques and enhanced gap junctional intercellular communication [32]. Our results show that the mechanism of action involves induction of liver-enriched transcription factors. We observed dramatic increases in several liver-enriched transcription factors in response to tRA treatment, which appear to coordinately shift the balance toward differentiation. Transcription factor expression is often dynamic, and its influence on any particular target gene is combinatorial. Nonetheless, the fact that a cohort of liverenriched transcription factors was unanimously induced is significant and indicative of a robust differentiation response. We have observed that differentiation function is induced by tRA both in HepG2 cells and in primary rat hepatocytes (Burley and Roth, submitted for publication). An agent such as tRA that can both control growth and promote differentiation is potentially beneficial for both anti-cancer applications and bioartifical liver devices.

\section{ACKNOWLEDGEMENTS}

This work was supported by an NSF CAREER Award (BES-0238617) and an NSF Metabolic Engineering grant (BES-0519563) to CMR and by an NIH Biotechnology Training Program Fellowship to MRB.

\section{REFERENCES}

[1] Yanagitani A, Yamada S, Yasui S, et al. Retinoic acid receptor alpha dominant negative form causes steatohepatitis and liver tumors in transgenic mice. Hepatology 2004; 40:366-375.

[2] Pinti M, Troiano L, Nasi M, Ferraresi R, Dobrucki J, Cossarizza A. Hepatoma HepG2 cells as a model for in vitro studies on mitochondrial toxicity of antiviral drugs: which correlation with the patient? J Biol Regul Homeost Agents 2003; 17:166-171.

[3] Knasmuller S, Mersch-Sundermann V, Kevekordes S, et al. Use of human-derived liver cell lines for the detection of environmental and dietary genotoxicants; current state of knowledge. Toxicology 2004; 198:315-328.

[4] Mersch-Sundermann V, Knasmuller S, Wu XJ, Darroudi F, Kassie F. Use of a human-derived liver cell line for the detection of cytoprotective, antigenotoxic and cogenotoxic agents. Toxicology 2004; 198:329-340.

[5] Cederbaum AI, Wu D, Mari M, Bai J. CYP2E1-dependent toxicity and oxidative stress in HepG2 cells. Free Radic Biol Med 2001; 31:1539-1543.

[6] Iyoda K, Sasaki Y, Horimoto M, et al. Involvement of the p38 mitogen-activated protein kinase cascade in hepatocellular carcinoma. Cancer 2003; 97:3017-3026.

[7] Koj A, Jura J. Complex analysis of genes involved in the inflammatory response: interleukin-1-induced differential transcriptome of cultured human hepatoma HepG2 cells. Acta Biochim Pol 2003; 50:573582 .

[8] Kurachi K, Zhang K, Ameri A, Huo J, Atoda H, Kurachi S. Genetic and molecular mechanisms of age regulation (homeostasis) of blood coagulation. IUBMB Life 2000; 49:189-196.

[9] Hongo T, Kajikawa M, Ishida S, et al. Three-dimensional highdensity culture of HepG2 cells in a 5-ml radial-flow bioreactor for construction of artificial liver. J Biosci Bioeng 2005; 99:237-244.

[10] Li R, Faria TN, Boehm M, Nabel EG, Gudas LJ. Retinoic acid causes cell growth arrest and an increase in p27 in F9 wild type but not in F9 retinoic acid receptor beta2 knockout cells. Exp Cell Res 2004; 294:290-300.
[11] Strickland S, Smith KK, Marotti KR. Hormonal induction of differentiation in teratocarcinoma stem cells: generation of parietal endoderm by retinoic acid and dibutyryl cAMP. Cell 1980; 21:347-355.

[12] Soprano KJ, Purev E, Vuocolo S, Soprano DR. Rb2/p130 and protein phosphatase 2A: key mediators of ovarian carcinoma cell growth suppression by all-trans retinoic acid. Oncogene 2006; 25:5315-5325.

[13] Jung HY, Park SH, Yoo YD, Kim JS, Kim YH. CDK2/4 regulate retinoic acid-induced G1 arrest in hepatocellular carcinoma cells. Hepatol Res 2005; 31:143-152.

[14] Chen Y, Dokmanovic M, Stein WD, Ardecky RJ, Roninson IB. Agonist and Antagonist of Retinoic Acid Receptors Cause Similar Changes in Gene Expression and Induce Senescence-like Growth Arrest in MCF-7 Breast Carcinoma Cells. Cancer Res 2006; 66:87498761.

[15] Alisi A, Leoni S, Piacentani A, Conti Devirgiliis L. Retinoic acid modulates the cell-cycle in fetal rat hepatocytes and HepG2 cells by regulating cyclin-cdk activities. Liver Int 2003; 23:179-186.

[16] Falasca L, Marcellini P, Ara C, Rufo A, Devirgiliis LC. Growth inhibition and induction of specific hepatic phenotype expression by retinoic acid in HEPG2 cells. Anticancer Res 1999; 19:3283-3292.

[17] Arce F, Gatjens-Boniche O, Vargas E, Valverde B, Diaz C. Apoptotic events induced by naturally occurring retinoids ATRA and 13cis retinoic acid on human hepatoma cell lines Hep3B and HepG2. Cancer Lett 2005; 229:271-281.

[18] Karlsson JO, Yarmush ML, Toner M. Interaction between heat shock and interleukin 6 stimulation in the acute-phase response of human hepatoma (HepG2) cells. Hepatology 1998; 28:994-1004.

[19] Liver Foundation Annual Report. Available from: http://www.liverfoundation.org

[20] Chamuleau RA, Deurholt T, Hoekstra R. Which are the right cells to be used in a bioartificial liver? Metab Brain Dis 2005; 20:327-335.

[21] Park JK, Lee DH. Bioartificial liver systems: current status and future perspective. J Biosci Bioeng 2005; 99:311-319.

[22] Corn PG, El-Deiry WS. Derangement of growth and differentiation control in oncogenesis. Bioessays 2002; 24:83-90.

[23] Yamashita Y, Shimada M, Harimoto N, et al. Histone deacetylase inhibitor trichostatin A induces cell-cycle arrest/apoptosis and hepatocyte differentiation in human hepatoma cells. Int J Cancer 2003; 103:572-576.

[24] Gamble MV, Blaner WS: Factors affecting blood levels of vitamin A. In: Livrea MA, Ed. Vitamin A and Retinoids: An Update of Biological Aspects and Clinical Applications. Boston, Birkhauser Verlag. 2000.

[25] Shin DJ, Odom DP, Scribner KB, Ghoshal S, McGrane MM. Retinoid regulation of the phosphoenolpyruvate carboxykinase gene in liver. Mol Cell Endocrinol 2002; 195:39-54.

[26] Lee IH, Lee JH, Lee MJ, Lee SY, Kim IS. Involvement of CCAAT/enhancer-binding protein alpha in haptoglobin gene expression by all-trans-retinoic acid. Biochem Biophys Res Commun 2002; 294:956-961.

[27] Mouthiers A, Mejdoubi N, Baillet A, Amelie P-A, Porquet D. Retinoids increase alpha-1 acid glycoprotein expression at the transcriptional level through two distinct DR1 retinoic acid responsive elements. Biochim Biophys Acta 2004; 1678:135-144.

[28] Bhatia SN, Balis UJ, Yarmush ML, Toner M. Probing heterotypic cell interactions: hepatocyte function in microfabricated co-cultures. J Biomater Sci Polym Ed 1998; 9:1137-1160.

[29] Dunn JC, Tompkins RG, Yarmush ML. Long-term in vitro function of adult hepatocytes in a collagen sandwich configuration. Biotechnol Prog 1991; 7:237-245.

[30] Ara C, Devirgiliis LC, Massimi M. Influence of retinoic acid on adhesion complexes in human hepatoma cells: a clue to its antiproliferative effects. Cell Commun Adhes 2004; 11:13-23.

[31] Brieva TA, Moghe PV. Exogenous cadherin microdisplay can interfere with endogenous signaling and reprogram gene expression in cultured hepatocytes. Biotechnol Bioeng 2004; 85:283-292.

[32] Ara C, Massimi M, Devirgiliis Conti L. Retinoic acid modulates gap junctional intercellular communication in hepatocytes and hepatoma cells. Cell Mol Life Sci 2002; 59:1758-1765. 